\title{
FORMULACIÓN Y ELABORACIÓN DE PRODUCTOS DE PANIFICACIÓN CON YACÓN (SMALLANTHUS SONCHIFOLIUS) COMO ENDULZANTE, PARA LA POBLACIÓN CON DEFICIENCIAS EN EL METABOLISMO DE LOS DISACÁRIDOS
}

\author{
FORMULATION AND ELABORATION OF BAKERY PRODUCTS \\ With YaCón SMALlanthus SONCHIfOLIUS AS SWEETENER, \\ FOR THE POPULATION WITH DEFICIENCIES IN THE \\ METABOLISM OF DISACCHARIDES.
}

\section{Flor Myriam Mejía Barragán}

Empresa Amerteck Ltda.

fmalimentos@gmail.com, orcid.org/0000-0003-0756-4880

Recibido: 23/09/2016 Aprobado: 30/10/2016

\section{RESUMEN}

El presente articulo se realizó utilizando el Yacón, del municipio de Machetá, departamento de Cundinamarca, en el Centro de Capacitación Marie Poussepin. Los resultados publicados de esta investigación descriptiva, que utiliza el método inductivo, tienen relación con los procesos de panificación utilizando Smallanthus sonchifolius. La orientación del documento se basó en la dificultad en la metabolización de los azúcares por algunas poblaciones, especialmente los diabéticos, en casos de obesidad o personas que utilicen productos bajos en calorias, lo que permite presentar esta nueva alternativa para la preparación de tortas, galletas y panes, logrando como resultados alimentos nutracéuticos, que en su composición conservan las cualidades nutricionales aptas para consumo masivo. Se desarrollaron tres formulaciones de cada producto, utilizando harinas de maíz, quinua y frutas como curuba y mora, típicas de la zona, endulzadas con yacón, por su bajo valor calórico $y$ altos contenidos en vitaminas y demás componentes nutricionales, siendo aceptados por los consumidores la galleta 2, la torta 3 y el pan 3 por sus caracteristicas organolépticas y fisicoquimicas. Los productos elaborados cumplen con las normas de inocuidad y las nuevas tendencias de estilos de vida saludables, lo que posibilita su introducción en mercados nacionales e internacionales, que están en busca de productos innovadores y ecológicos, como alternativas para los diferentes tipos de población que están entrando en la filosofía wellness.

Palabras clave: nutracéuticos, oligosacáridos, prebióticos, wellness, yacón. 
Formulación y elaboración de productos de panificación con yacón (Smallanthus sonchifolius)

como endulzante, para la población con deficiencias en el metabolismo de los disacáridos

\section{Abstract}

The present article was made using the yacón of the municipality of Macheta Department of Cundinamarca at the Marie Poussepin Training Center. The published results of this descriptive research that uses the inductive method, is related to the baking processes using Smallanthus sonchifolius The orientation of the document is based on the difficulty in the metabolization of sugars by some populations, especially diabetics, in cases of obesity or people using low calorie products, giving rise to presenting this new alternative to start with the formulation and development of cakes, biscuits and breads, resulting in nutraceutical foods, which in their composition retain the nutritional qualities suitable for mass consumption. Three formulations of each product were developed using maize, quinoa and fruits like curuba and blackberry, typical of the area, sweetened with yacon, because of their low caloric value and high contents of vitamins and other nutritional components, being accepted by consumers the cookie 2, cake 3 and bread 3 for their organoleptic and physicochemical characteristics. The processed products comply with the safety standards and the new trend of healthy lifestyles, which impact the national and international markets that are looking for innovative and ecological products, as alternatives for the different types of population entering the wellness philosophy.

Key words: nutraceuticals, oligosaccharides, prebiotics, wellness, yacón.

\section{INTRODUCCIÓN}

Los antiguos pobladores andinos han utilizado las raíces de yacón desde tiempos inmemoriales, y en la actualidad se puede observar, que, desde las últimas décadas, ha aumentado su consumo por el alto contenido de fructooligosacáridos (FOS), lo que permite explicar el efecto pre-biótico de los extractos del fruto, debido a que los azúcares presentes no son hidrolizados por las enzimas digestivas de los humanos, pero si por las bacterias del tracto intestinal (Pedreschi et al. 2003). Además, contienen antioxidantes naturales de importancia para la salud humana como son compuestos fenólicos derivados del ácido clorogénico y cafeico (Seminario, Manrique \& Valderrama 2003).

En el mundo la diabetes mellitus, que consiste en un desorden metabólico, afecta aproximadamente a 143 millones de personas, y según la Organización Mundial de la Salud, se espera que para el año 2030 el número de pacientes se aproxime a 300 millones (Boyle, et al. 2001; Tamayo 2005; Tiwari \& Rao 2002).
Teniendo en cuenta lo anterior, este estudio fue iniciado conociendo las dificultades en el metabolismo de los azúcares por la población colombiana, es así, como el Ministerio de Salud y Protección Social (2017) establece que más de dos millones de personas pueden tener diabetes y no saberlo, esta enfermedad se ha convertido en epidemia y anualmente cobra la vida de 20.000 personas dando lugar a un problema de salud pública. Además el país cuenta con 3.3 millones de personas con esta enfermedad, de las cuales 2.2 millones están diagnosticadas.

El objetivo del presente estudio consiste en desarrollar productos (Manrique, Párraga y Hermann 2005) de panificación utilizando el yacón, fruto que se cultiva en América Central y Sur América, y por su sabor dulce es considerado una fruta, a pesar de ser una raíz tuberosa. Las propiedades del yacón son conocidas desde la antigüedad andina, aunque en los últimos ańos se han revaluado sus características fisicoquímicas y nutricionales, por las diversas investigaciones y por la aceptación de los consumidores. 
Las formulaciones (Margalef et al. 2013), para los productos de panificación tortas, galletas y panes son desarrollados y endulzados a partir del yacón, utilizando otras materias primas como curuba, mora, quinua y maíz, que por sus altos contenidos nutricionales dan como resultado productos innovadores nutracéuticos.

\section{MARCO TEÓRICO}

El yacón (Gónzalez et al. 2016; Lachman, Fernández y Orsak 2003) puede ser una alternativa de solución por poseer carbohidratos tipo FOS. Este tubérculo es una especie de la familia Asteraceae y su nombre científico es Smallanthus sonchifolius, es una planta herbácea perenne, mide de $1-3$ metros de alto dependiendo de su especie. Las raíces fibrosas son delgadas y su función es la fijación de la planta al suelo, absorción de agua y nutrientes, para lo cual se debe determinar la huella hídrica (Díaz, Lizarazo \& Torres 2016; Sánchez, Villareal \& Torres 2014), mientras que las raíces reservantes son engrosadas, fusiformes y ovaladas de color púrpura, blanco o crema generalmente.

La cosecha de la raíz (Lachman, Fernández \& Orsak 2003) se realiza entre los $6-8$ meses, dependiendo de la variedad. Los indicios de madurez se presentan cuando la planta se torna amarillenta, hay caída de las hojas y finalización de la floración.

La mayoría de los tubérculos (Yan et al. 1999) almacenan los carbohidratos en forma de almidón, pero el yacón los almacena esencialmente como fructooligosacáridos (FOS), una clase especial de azúcares que tienen pocas calorías y no elevan los niveles de glucosa en la sangre, propiedad que ha llevado a éste fruto al mercado de los productos dietéticos y alimentos funcionales (López 2007).

Los fructooligosacáridos (FOS) o fructanos (Gordillo 2013), son oligopolímeros formados por la unión de una cadena $\beta-2,1$ de D-fructosa (F) a una unidad de D-glucosa (G). Según evidencia científica (Sánchez \& Genta 2007; Sangeetha \& Prapulla 2005) hay otras propiedades de los FOS, que han demostrado que pueden disminuir los niveles de colesterol y triglicéridos, incrementar la asimilación del calcio en los huesos, fortalecer el sistema inmunológico y restaurar la flora intestinal, cuando son consumidos en forma natural en verduras y frutas. Si los FOS se añaden en forma de aditivos alimentarios como productos refinados y más concentrados, pueden producir una sobrecarga en el intestino generando hinchazón, flatulencia y algunas incomodidades en el organismo (Alfaro 2004).

El consumo de fructooligosacáridos son los mejores prebióticos conocidos, demostrando el efecto bifidogénico al no ser digerido por las enzimas intestinales, y llegando al colon donde se fermentan de forma anaerobia por la microflora, incrementando la población de bacterias benéficas, impidiendo el crecimiento de organismos patógenos que son responsables de la producción de toxinas y compuestos que pueden ser potencialmente cancerígenos (Madrigal \& Sangronis 2007).

Variados estudios muestran (Madrigal \& Sangronis 2007) que el consumo de fructooligosacáridos producen una reducción importante en los niveles de azúcar y grasas en la sangre, inhibiendo la lipogénesis hepática, los riesgos de arterioesclerosis, en algunos casos normalizando la presión arterial y mejorando el metabolismo de las personas que sufren problemas cardiovasculares, diabetes y colesterol alto.

Experimentalmente (Bruggebcate et al. 2006) se ha demostrado que la asociación de la inulina más oligofructosa presente en el yacón puede prevenir la colitis al modificar la microflora intestinal actuando como un prebiótico.

Un estudio realizado sobre la valoración (Huaycho et al. 2016; Institute for Food Technologist 2013; Lachman, Fernández \& Orsak 2003) de la raíz de yacón, establece las composiciones porcentuales de los principales azúcares que forman los carbohidratos solubles de dos variedades de yacón blanco y morado como se puede observar en la Fig. 1. 
Formulación y elaboración de productos de panificación con yacón (Smallanthus sonchifolius) como endulzante, para la población con deficiencias en el metabolismo de los disacáridos

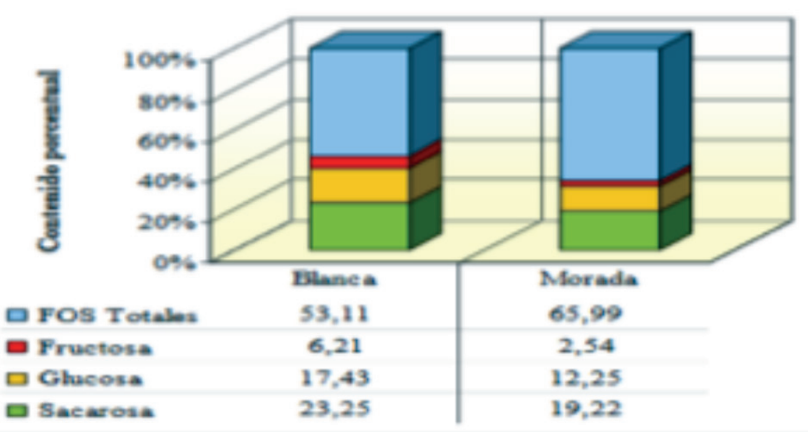

Fig. 1. Tipos de azúcares en variedades de yacón. Fuente: Huaycho H.; Aruquipa R., IFT., Lachman J., 2016

La composición nutricional (Margalef et al. 2013) del yacón está constituida por azucares FOS, que también son ingredientes prebióticos con cadenas cortas de fructosa unidas a una molécula de glucosa y carbohidratos con azúcares simples, con bajo contenido calórico que puede ser metabolizado directamente por el tracto digestivo. En la Tabla 1 se presenta la composición del yacón en 100 gramos de raíz fresca sin cáscara.

\section{TABLA 1}

Composición nutricional del yacón

\begin{tabular}{|l|c|}
\hline \multicolumn{1}{|c|}{ COMPUESTO } & RANGO \\
\hline Energía Kcal. & $14.0-54.0$ \\
\hline Agua g. & $86.6-90.0$ \\
\hline Proteína g. & $0.1-0.5$ \\
\hline Grasa g. & $0.3-12.6$ \\
\hline Carbohidratos g. & $12.5-23.65$ \\
\hline Oligofructosa (OF) g. & $6.0-19.0$ \\
\hline Azúcares simples* g. & $1.5-4.0$ \\
\hline Fibra g. & $0.5-4.1$ \\
\hline Ceniza g. & $0.3-0.67$ \\
\hline Calcio mg. & $6.0-23.0$ \\
\hline Fósforo mg. & $21-25.4$ \\
\hline Hierro mg. & $0.3-042$ \\
\hline Potasio mg. & $185.0-295.0$ \\
\hline Retinol mg. & $12.0-12.8$ \\
\hline Tiamina mg. & $0.02-0.03$ \\
\hline Riboflavina mg. & $0.11-013$ \\
\hline Niacina mg. & $0.34-0.36$ \\
\hline Ácido ascórbico mg. & -13.6 \\
\hline & \\
\hline
\end{tabular}

* Incluye sacarosa, glucosa y fructosa.

Fuente: Margalef M.I.,Gómez M.H.,Jiménes M.J., 2013.
Los análisis químicos del yacón (Institute for Food Technologist 2013; Lachman, Fernández \& Orsak 2003) comparan los resultados en porcentaje con base seca y base húmeda, que permite establecer las concentraciones reales del fruto en cuanto a sus componentes, con el fin de establecer los factores que pueden variar, en los procesos de elaboración de productos que requieren de aplicación de procesos térmicos. La Tabla 2 establece las variaciones en valores por componentes.

TABla 2

Análisis químico de la raíz de yacón

\begin{tabular}{|l|c|c|}
\hline COMPONENTE & $\begin{array}{c}\text { VALORES } \\
\text { YACÓN BASE } \\
\text { SECA \% }\end{array}$ & $\begin{array}{c}\text { VALORES } \\
\text { YACÓN BASE } \\
\text { HÚMEDA \% }\end{array}$ \\
\hline Humedad & $83.56-91.34$ & $13.08-13.16$ \\
\hline Proteína & $2.59-3.12$ & $17.88-18.12$ \\
\hline Fibra cruda & $16.91-18.39$ & $7.54-7.87$ \\
\hline $\begin{array}{l}\text { Carbohidratos } \\
\text { solubles totales }\end{array}$ & $71.80-75.64$ & $35.68-36.70$ \\
\hline Almidón & $2.61-2.98$ & ---- \\
\hline Cenizas & $5.10-5.78$ & $1.88-1.96$ \\
\hline Grasas & $0.95-1.03$ & $11.55-11.86$ \\
\hline
\end{tabular}

Fuente: Institute of Food Technologist (IFT),2013, Lachman J., Fernandez E.C., 2013

\section{DISEÑO EXPERIMENTAL}

En el desarrollo del documento se utiliza el diseño de investigación descriptiva (Takaneka et al. 2003) y aplicada, donde se especifican los parámetros fisicoquímicos, microbiológicos y sensoriales del producto final; $y$ se practican las técnicas de elaboración del producto con beneficios nutricionales para el consumidor.

Se utiliza el método inductivo, a partir de una observación real de la población estudiantil del Centro de Capacitación Rural Marie Poussepin, ubicado en el municipio de Machetá, complementándolo con el análisis de los datos recopilados, estableciendo las causas y efectos reales. 
Para la selección de la muestra se utiliza la ecuación para población finita con un nivel de confianza del $95 \%$ y un porcentaje de error del $5 \%$.

En las pruebas de aceptación y evaluación sensorial de los productos se aplicó la Escala Likert o Hedónica (Chen, Resurreción \& Paguio 1996) formulario de nueve puntos como se relaciona en la Tabla 3 con las siguientes apreciaciones:

TABla 3

Formato escala hedónica de nueve puntos

\begin{tabular}{|l|c|}
\multicolumn{1}{|c|}{ OBSERVACIÓN } & VALOR \\
\hline Gusté extremadamente & 9 \\
\hline Gusté mucho & 8 \\
\hline Gusté moderadamente & 7 \\
\hline Gusté ligeramente & 6 \\
\hline Me es indiferente (ni me gustó ni me disgustó) & 5 \\
\hline Disgusté ligeramente & 4 \\
\hline Disgusté moderadamente & 3 \\
\hline Disgusté mucho & 2 \\
\hline Disgusté extremadamente & 1 \\
\hline
\end{tabular}

Fuente: Chen A.W., Resurrección A.V.A. y Paguio L.P., 1999

\section{Materiales y MéTodos}

Para el estudio se utilizó la raíz procedente del municipio de Machetá (Cundinamarca), variedad amarilla de yacón común.

\section{1) Proceso de obtención del yacón como materia} prima: recolección de los frutos cada dos meses para establecer los cambios físicos y químicos, con el fin de seleccionar las condiciones óptimas para la caracterización del tubérculo.

2) Almacenamiento de raíces: se almacenaron inicialmente en refrigeración a una temperatura entre 4 - 7 grados centígrados sin ningún proceso de lavado, para asegurar la composición del fruto. Posteriormente fueron lavadas, desinfectadas, peladas y escaldadas para el proceso de producción aplicando una solución ácido ascórbico en un rango entre $0.4-0.5$ por ciento.

3) Características fisicoquímicas: los métodos analíticos cuantitativos de la AOAC (American Official Association of Analytical Chemists), Humedad (10.102), proteína (14.026), grasa (10.142), fibra(10.145), cenizas (10.144) y carbohidratos por diferencia.

4) Análisis microbiológico: para los productos finales se realizaron los siguientes análisis microbiológicos: recuento de aerobios mesófilos (Norma NTC 4519), recuento de mohos (Norma NTC 4132), Recuento de levaduras (Norma NTC 4132), número más probable de coliformes totales por gramo (Norma NTC 4516), número más probable de coliformes fecales $45^{\circ} \mathrm{C}$ (norma NTC 4516) y estafilococo coaguloso positivo (Norma NTC 7479).

5) Pruebas sensoriales: se desarrollaron nueve (9) formulaciones, tres (3) para cada tipo de producto, a los cuales se aplicó el formulario de nueve (9) puntos de la Escala de Likert o Hedónica.

\section{Resultados}

1) Caracterización de materia prima: el yacón un recurso promisorio (Mansilla et al. 2010; Quintana, Gelvez \& Mendoza 2014); el utilizado en el estudio, proviene de las fincas vecinas al Centro de Capacitación Marie Posussepin ubicado en el municipio de Machetá, que cuenta con una planta de producción artesanal.

Para la etapa inicial del proyecto se tomaron como base las referencias (González et al. 2016; Mansilla et al. 2010; Valderrama \& Manrique 2003; Valderrama 2005) en la caracterización de la materia prima, realizando el seguimiento al cultivo cada dos (2) meses, con el fin de establecer los cambios en el crecimiento del fruto y seleccionar la etapa óptima para el proceso de producción como se observa en la Fig. 2. 
Formulación y elaboración de productos de panificación con yacón (Smallanthus sonchifolius)

como endulzante, para la población con deficiencias en el metabolismo de los disacáridos

\begin{tabular}{|l|c|c|c|}
\hline Batata Rizopo inicial & Siembra de Batata & Forma de Tallo & Cultivo 2 meses \\
\hline Cultivo 4 meses & Cultivo 5 meses & Cultivo 6 meses & Cultivo 8 meses \\
\hline
\end{tabular}

Dos meses Fase I: Determinación de los contenidos de almidón en el fruto utilizando una solución de lugol al $1 \%$, no se observa ningún cambio, la pulpa mantiene un color azul - violeta intenso.

Cuatro meses Fase II: Se inicia la conversión del almidón en azucares, lo cual se observa en el gráfico con la presencia de espacios blancos.
Seis meses Fase III : Se inicia la reducción de almidón de forma más pronunciada, observándose en el centro de la pulpa una estrella, indicadora de la formación de mayor cantidad de azúcares.

Ocho meses Fase IV: La reacción muestra la conversión del almidón en azucares en la pulpa, solo se observa presencia de almidón en la cáscara del fruto.

Fig. 2 Seguimiento del cultivo de yacón.

Fuente: seguimiento de cultivo en campo por Autor

2). Prueba del lugol al 1\%: para la determinación del almidón producido en el fruto durante el seguimiento del cultivo, se utilizó la prueba de lugol, la cual al entrar en contacto con la pulpa de yacón cambia a color azul - violeta característico, indicando el momento en el cual el almidón se va convirtiendo en azúcares, lo cual se determinó con el cambio de coloración hasta blanco como se puede observar en la fig. 3 .

3). Análisis fisicoquímico del yacón: el resultado del análisis proximal del yacón maduro utilizado como materia prima para el proceso de producción de panificación se reporta en la Tabla 4.
TABla 4

Análisis proximal del yacón con base seca (Lachman, Fernandez \& Orsak 2003)

\begin{tabular}{|l|c|}
\hline \multicolumn{1}{|c|}{ COMPONENTE } & RANGOS DE VALOR \\
\hline Energético Kcal & $363.9-393.7$ \\
\hline Humedad \% & $81.2-83.5$ \\
\hline Grasa \% & $0.17-0.24$ \\
\hline Proteína \% & $2.6-2.8$ \\
\hline Carbohidratos \% & $88.0-93.2$ \\
\hline Fibra bruta \% & $3.2-4.1$ \\
\hline Cenizas \% & $2.5-3.2$ \\
\hline Sólidos solubles ( ${ }^{\circ}$ Brix) & $14.1-16.5$ \\
\hline pH & $6.3-6.7$ \\
\hline Acidez (ácido cítrico) & $0.29-0.36$ \\
\hline Aw & $0.83-0.87$ \\
\hline
\end{tabular}

Fuente: Lachman J., Fernandez E.C., Orsak M., 2003 
4). Características físicas del yacón: el resultado de la observación del fruto maduro, lavado y pelado es presentado en la Tabla 5.

\section{TABLA 5}

Características físicas del yacón.

\begin{tabular}{|l|c|}
\hline \multicolumn{1}{|c|}{ COMPONENTE } & CARACTERÍSTICA \\
\hline Aspecto & Granuloso - estriado \\
\hline Color & Blanco - amarillo \\
\hline Olor & Característico \\
\hline Sabor & Dulce \\
\hline
\end{tabular}

Fuente: Autor

5). Formulaciones: para la formulación y preparación de las muestras se tuvo en cuenta el procedimiento propuesto para una barra con harina de yacón (Valdez, Margalef \& Gómez 2003). Se tomaron como base las composiciones de las diferentes harinas y el yacón, dando como resultado productos que se pueden clasificar entre los alimentos nutracéuticos, como es el caso en éste proyecto, utilizando la goma xantana para aumentar la viscosidad en panes y tortas, haciendo la masa más consistente, con aumento de la elasticidad de la mezcla mejorando las texturas de los productos finales.
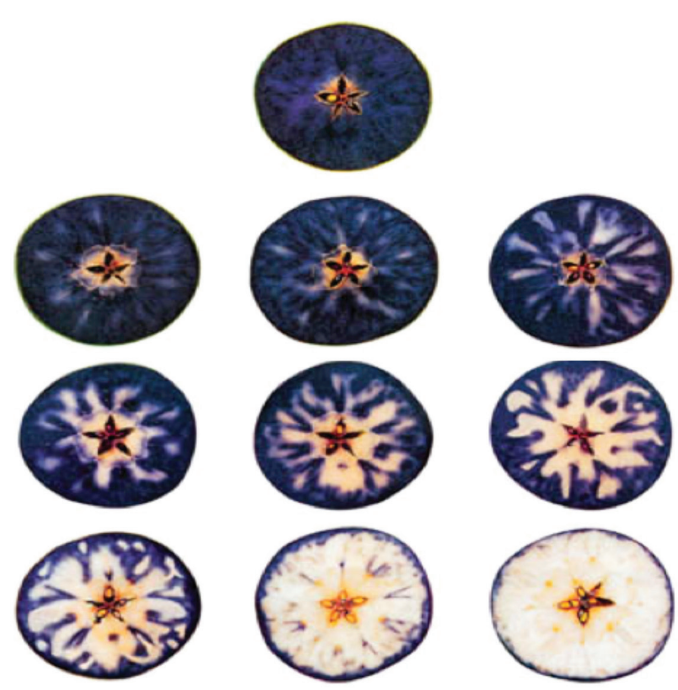

Fig. 3 Seguimiento de la maduración del yacón.

Fuente: seguimiento de la maduración del yacón en campo por Autor

Para todas las formulaciones se mezclaron las harinas de maíz porva y quinua dulce en diferentes proporciones para asegurar la homogeneidad en la mezcla.

Para el yacón se desarrollaron los almibares de los dulces sin adición de agua, con el fin de utilizar el agua presente en la composición de los frutos, rectificando el dulzor final con sucralosa. La Tabla 6 muestra las composiciones de galletas y panes respectivamente.

TABla 6

Formulaciones de galletas y panes

\begin{tabular}{|l|c|c|c|l|c|c|c|}
\hline COMPONENTES & $\begin{array}{c}\text { GALLETA } \\
\mathbf{1}(\mathbf{\%})\end{array}$ & $\begin{array}{c}\text { GALLETA } \\
\mathbf{2}(\mathbf{\%})\end{array}$ & $\begin{array}{c}\text { GALLETA } \\
\mathbf{3}(\mathbf{\%})\end{array}$ & COMPONENTES & $\begin{array}{c}\text { PAN 1 } \\
\mathbf{( \% )}\end{array}$ & $\begin{array}{c}\text { PAN 2 } \\
(\mathbf{\%})\end{array}$ & $\begin{array}{c}\text { PAN 3 } \\
\mathbf{( \% )}\end{array}$ \\
\hline Harina maíz + quinua & 45 & 50 & 55 & Harina maíz + quinua & 50.6 & 40.6 & 35.5 \\
\hline Grasa vegetal & 20 & 25 & 25 & Agua & 20.6 & 10.6 & 10.0 \\
\hline Yacón + moras & 34 & - & - & Yacón & 9.1 & 15.5 & 21.0 \\
\hline Yacón + curubas + moras & - & 24 & - & Margarina & 9.1 & 9.1 & 9.1 \\
\hline Yacón + curuba & - & - & 18 & Huevo & 10.5 & 12.8 & 13.8 \\
\hline Sucralosa (rectificar) & 1.0 & 1.0 & - & Goma Xanta + sucralosa & 9.1 & 11.4 & 10.6 \\
\hline
\end{tabular}

6). Análisis de aceptación de productos: para determinar la aceptabilidad y evaluación sensorial de los productos se utilizó la Escala Likert o Hedónica formulario de nueve puntos, siendo el valor de mayor aceptación 9 y el de menor 1 (Chen, Resurrección \& Paguio 1996).
El cálculo de la muestra arrojó un resultado de 60 personas con un nivel de confianza del $95 \%$ y un error correspondiente al 5\%. Los participantes no entrenados a los cuales se les aplicó el formato oscilaron en edades entre 15 - 35 años, siendo realizada en el Centro de Capacitación Rural Marie Poussepin ubicado en el municipio de Machetá. 
Formulación y elaboración de productos de panificación con yacón (Smallanthus sonchifolius)

como endulzante, para la población con deficiencias en el metabolismo de los disacáridos

Con los resultados de aceptación se elaboró un diagrama de sectores para los diferentes tipos de productos elaborados, como se puede observar en la Fig. 4.
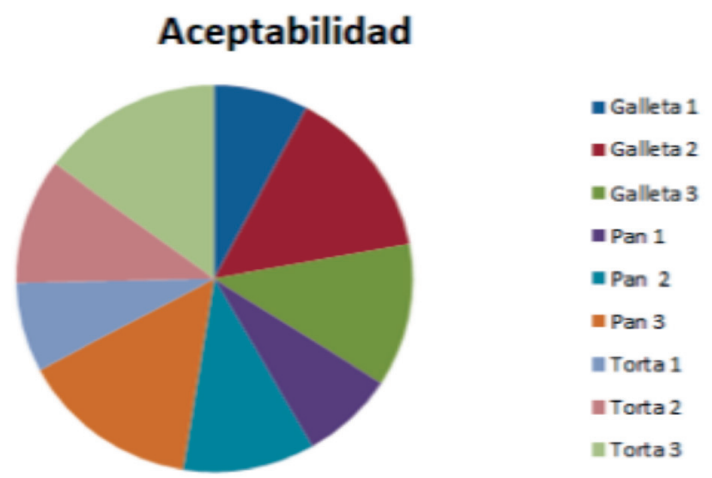

Fig. 4. Resultados de aceptación para cada producto. Fuente: autor
Los promedios establecidos para cada una de las muestras seleccionadas evalúo el grado de satisfacción de cada producto, encontrando en los rango entre 6.86 - 7.06, con mediana entre 6.0 - 7.0, los valores de me gusta ligeramente y moderadamente, estableciendo que ninguna de las muestras seleccionadas fue rechazada en su totalidad, como se puede constatar en los valores mínimos con rangos entre 1 y 3 para cada producto evaluado. El coeficiente de correlación varía entre $0.75-0.91$ mostrando que hay una correspondencia entre las variables, presentando los resultados en la Tabla 7.

TABLA 7

Resultados estadísticos de aceptación para los productos elaborados

\begin{tabular}{|c|c|c|c|c|c|c|c|c|c|}
\hline DATOS & $\underset{1}{\text { GALLETA }}$ & $\underset{2}{\text { GALLETA }}$ & $\begin{array}{c}\text { GALLE- } \\
\text { TA } \\
3\end{array}$ & $\begin{array}{c}\text { PAN } \\
1\end{array}$ & $\begin{array}{c}\text { PAN } \\
2\end{array}$ & $\begin{array}{c}\text { PAN } \\
3\end{array}$ & $\begin{array}{c}\text { TORTA } \\
1\end{array}$ & $\begin{array}{c}\text { TORTA } \\
2\end{array}$ & $\begin{array}{c}\text { TORTA } \\
3\end{array}$ \\
\hline$\sum \mathrm{x}$ & 220 & 412 & 341 & 220 & 306 & 418 & 214 & 300 & 424 \\
\hline Promedio & 3.66 & 6.86 & 5.68 & 3.66 & 5.10 & 6.96 & 3.56 & 5.00 & 7.06 \\
\hline$\sigma$ muestra & 5.65 & 5.66 & 4.24 & 7.33 & 2.97 & 3.49 & 7.50 & 3.29 & 3.38 \\
\hline Coef. correlación & 0.99 & 0.75 & 0.85 & 0.85 & 0.79 & 0.89 & 0.89 & 0.76 & 0.91 \\
\hline Mínimo & 1 & 1 & 3 & 1 & 2 & 3 & 1 & 2 & 3 \\
\hline Máximo & 9 & 9 & 9 & 9 & 9 & 9 & 9 & 9 & 9 \\
\hline Mediana & 3 & 7 & 6 & 5.00 & 5.50 & 6.00 & 3 & 5 & 7 \\
\hline$\sigma$ población & 7.11 & 4.37 & 2.91 & 16 & 12.25 & 9.0 & 7.37 & 3.23 & 3.32 \\
\hline
\end{tabular}

Fuente: propia

7) Análisis proximales y microbiológicos: a partir de los resultados de los análisis proximales se calculó la composición nutricional de cada uno de los productos seleccionados y con los resultados microbiológicos, que se muestra en la Tabla 8 se puede constatar el cumplimiento de los requisitos de inocuidad para productos alimenticios (Bernal 2014). Otra Técnica de eliminación de contaminantes biológicos sería recomendable la aplicación de ultrasonido para asegurar la inocuidad en un estudio posterior (Delgado 2016). 
TABLA 8

Resultados del análisis microbiológico para cada producto seleccionado

\begin{tabular}{|l|c|c|c|c|c|}
\hline \multicolumn{1}{|c|}{ PARÁMETRO } & MÉTODO & GALLETA 2 & PAN 3 & TORTA 3 & $\begin{array}{c}\text { VALOR } \\
\text { ACEPTADO }\end{array}$ \\
\hline Recuento bacterias de aerobias mesofilas & Invima & $<10 \mathrm{UFC} / \mathrm{G}$ & $<10 \mathrm{UFC} / \mathrm{G}$ & $<10 \mathrm{UFC} / \mathrm{G}$ & $<10000 \mathrm{UFC} / \mathrm{G}$ \\
\hline Coliforme total & Invima & $<3 / \mathrm{G}$ & $<3 / \mathrm{G}$ & $<3 / \mathrm{G}$ & $<9 / \mathrm{G}$ \\
\hline Coliforme fecal & Invima & $<1 / \mathrm{G}$ & $<1 / \mathrm{G}$ & $<1 / \mathrm{G}$ & $<3 / \mathrm{G}$ \\
\hline Recuento hongos y levaduras & Invima & $\begin{array}{c}\mathrm{H}:<10 \mathrm{~L}:<10 \\
\mathrm{UFC} / \mathrm{G}\end{array}$ & $\begin{array}{c}\mathrm{H}:<10 \mathrm{~L}:< \\
10 \mathrm{UFC} / \mathrm{G}\end{array}$ & $\begin{array}{c}\mathrm{H}:<10 \mathrm{UFC} / \mathrm{G} \\
<10\end{array}$ & $200 \mathrm{UFC} / \mathrm{G}$ \\
\hline Recuento de estafilococo coagulasa positiva & Invima & $<30 \mathrm{UFC} / \mathrm{G}$ & $<30 \mathrm{UFC} / \mathrm{G}$ & $<30 \mathrm{UFC} / \mathrm{G}$ & $<100 \mathrm{UFC} / \mathrm{G}$ \\
\hline Calidad microbiológica & & & & Aceptado \\
\hline
\end{tabular}

Fuente: Resultados de Laboratorio Nulab 2017

8) Análisis sensorial: establecidas y validadas las formulaciones de la galleta 2 , pan 3 y torta 4 a partir de la aplicación de la escala de Likert o Hedónica de 9 puntos se presentan a continuación los resultados obtenidos de la evaluación para las características de sabor, color, olor, textura en cada uno de los productos, en la Tabla 9, y los porcentajes por producto y la comparación de cada uno en la Fig. 5.

\section{TABLA 9}

Resultados en porcentaje de cada característica sensorial de los productos seleccionados.

\begin{tabular}{|l|c|c|c|c|}
\hline & $\begin{array}{c}\text { SABOR } \\
(\%)\end{array}$ & $\begin{array}{c}\text { COLOR } \\
(\%)\end{array}$ & $\begin{array}{c}\text { OLOR } \\
(\%)\end{array}$ & $\begin{array}{c}\text { TEXTURA } \\
(\%)\end{array}$ \\
\hline Pan 1 & 10 & 5 & 10 & 9 \\
\hline Pan 2 & 30 & 25 & 30 & 11 \\
\hline Pan 3 & 60 & 70 & 60 & 80 \\
\hline Galleta 1 & 10 & 5 & 8 & 15 \\
\hline Galleta 2 & 70 & 80 & 80 & 60 \\
\hline Galleta 3 & 20 & 15 & 12 & 25 \\
\hline Torta 1 & 6 & 10 & 5 & 10 \\
\hline Torta 2 & 14 & 20 & 15 & 20 \\
\hline Torta 3 & 80 & 70 & 80 & 70 \\
\hline
\end{tabular}

Fuente: autor
El pan 3 fue el de mayor aceptación entre los degustadores, con un contenido de harina de maíz quinua del $35.5 \%$ y de yacón del $21 \%$, por su agradable sabor dulce además de contar con las características de color amarillento jaspeado, corteza jaspeada uniforme y brillante, olor agradable, sabor característico, textura de la corteza fina y miga suave al tacto.

Los panes 1 y 2 presentaron evaluaciones más baja de aceptación en cuanto a la textura, puesto que su consistencia fue muy harinosa y se desmoronó con mucha facilidad, dando resequedad en la boca no característica para este tipo de productos. Para productos sería recomendable ensayar aplicando una técnica de fermentación (Quitan, Gelvez \& Mendoza 2014).

Mediante la evaluación sensorial se estableció que la galleta con mayor aceptación fue la No. 2 que contiene $50 \%$ de harina de maíz - quinua, con textura y crocancia característica, contenido del $24 \%$ de yacón - mora - curuba, sabor de mayor preferencia entre los degustadores.

Con relación a las galletas 1 y 3 el color obtuvo mucho rechazo por parte de los evaluadores, porque las frutas utilizadas dan al producto final una coloración oscura no homogénea en el caso de la mora, pero en el caso de la curuba el color jaspeado resultante no fue del gusto de los consumidores, lo cual se contrasta con el color mora - curuba de la galleta 2 que obtuvo la mayor aceptación. 
Formulación y elaboración de productos de panificación con yacón (Smallanthus sonchifolius)

como endulzante, para la población con deficiencias en el metabolismo de los disacáridos

Una prueba recomendada para mejorar el sabor y color de los diferentes productos sería aplicar una técnica de deshidratación de frutas (Giraldo, Vargas $\&$ Gil 2016).

Para la torta No.3 con composición de maíz - quinua del $48 \%$ - yacón del $15.7 \%$ con cubierta curuba-mora fue la de mayor aceptación, con característica de consistencia semi esponjosa, color interno jaspeado, corteza suave y apariencia fresca, sabor dulce acentuado agradable, especialmente por la mezcla de almibares de frutas mora y curuba.
La baja aceptabilidad de las tortas 1 y 2 se debió a la falta de consistencia de las mezclas de harina y quinua que dio como resultado una textura seca, no típica de este tipo de productos, aunque fueron muy bien valoradas las cubiertas tanto de mora como de curuba.

9) Cotejo de productos: con el fin de establecer los cambios en la composición nutricional de los productos elaborados se desarrollaron formulaciones de las muestras seleccionadas, galleta 2 , pan 3 y torta 3 cambiando las harinas maíz porva y quinua por harina de trigo y el yacón por azúcar en los mismos porcentajes, los resultados se observan en la Tabla 10.

TABla 10

Diferencias de composición nutricional en 100 gramos de porción comestible entre producto contraste y producto seleccionado

\begin{tabular}{|l|c|c|c|c|c|c|}
\hline PARÁMETRO & $\begin{array}{c}\text { GALLETA } \\
\text { CONTRASTE }\end{array}$ & $\begin{array}{c}\text { GALLETA } \\
\mathbf{2}\end{array}$ & $\begin{array}{c}\text { PAN } \\
\text { CONTRASTE }\end{array}$ & $\begin{array}{c}\text { PAN } \\
\mathbf{3}\end{array}$ & $\begin{array}{c}\text { TORTA } \\
\text { CONTRASTE }\end{array}$ & $\begin{array}{c}\text { TORTA } \\
\mathbf{2}\end{array}$ \\
\hline Energía Kcal & 442.41 & 348.60 & 286,50 & 258,60 & 404.10 & 308.02 \\
\hline Humedad \% & 2.30 & 3.84 & 9.70 & 21.83 & 22.61 & 30.37 \\
\hline Carbohidratos\% & 77.50 & 73.19 & 59.41 & 64.65 & 66.10 & 77.00 \\
\hline Proteína \% & 7.90 & 8.56 & 7.52 & 8.39 & 8.01 & 5.65 \\
\hline Grasa \% & 11.20 & 5.23 & 2.10 & 3.89 & 11.98 & 3.95 \\
\hline Fibra Cruda \% & 0.50 & 2.50 & 0.72 & 2.98 & 0.10 & 4.70 \\
\hline Cenizas \% & 1.40 & 1.74 & 1.50 & 3.44 & 1.80 & 2.49 \\
\hline
\end{tabular}

Fuente: Resultados de Laboratorio Nulab

Las diferencias nutricionales en el pan son aún más notorias con respecto a la composición en proteína, fibra y cenizas. Es importante señalar que hay un aumento en los contenidos de agua y grasa debido a que la quinua requiere para la mezcla mayor cantidad de estos componentes para la elaboración de la masa.

Las diferencias nutricionales de las tortas, en una relación de harinas 2:2:2 para torta 3, seleccionada en la evaluación sensorial, con respecto a la torta con harina de trigo y azúcar, muestra un valor mucho menor en cuanto a composición proteica, indicando que se requiere una reformulación de la mezcla para mejorar los niveles en este componente, pero en cambio, si se presenta un aumento casi de tres veces en el valor de la fibra, y de dos veces con relación a la composición de cenizas en el producto final.

Según los resultados anteriores se puede observar que la adición de quinua en el producto incrementa el contenido de proteína y la adición de harinas y leche en las formulaciones asegura que fue el mejor comportamiento en la formulación con resultado del $48 \%$.

Con la mezcla propuesta 2:2.2 se presentaron muchas dificultades para el logro de la masa que permitiera la 
textura esponjosa característica de este tipo de producto. Lo mismo sucedió con los productos torta 1 y 2 , por lo cual se seleccionó el porcentaje de $48 \%$ para todas las formulaciones que fue el de mejor comportamiento.

Una dificultad que presentó el proyecto fue la de asegurar la misma variedad de quinua en la elaboración de los productos finales, probablemente, a esta condición se deban los rangos en la información de los análisis proximales de los diferentes productos, dado que el yacón siempre fue de la misma variedad lo mismo que el maíz porva.

Se recomendaría para una nueva etapa de este estudia la aplicación de un control predictivo con el fin de iniciar las pruebas de producción industrial (Sendaya 2013), además de ensayar con cubiertas y películas con aditivos bioactivos para la conservación de la fruta ( $\mathrm{Si}$ tonio \& Menegalli 2013).

\section{ConClusiones}

Las galletas con composición en harinas de maíz porva, quinua $50 \%$, yacón con porcentaje del $24 \%$ almíbar curuba - mora, fue seleccionada en la evaluación sensorial como la de mayor aceptación dentro de la población que participó en la degustación.

El pan 3 que contenía en su composición el 21\% de yacón y un $35.5 \%$ en porcentaje de harinas, fue el de mejor comportamiento en cuanto al desarrollo de las características típicas de un pan dulce, con respecto a las otras pruebas, que presentaron cambios importantes en la composición de la miga y la presentación final del producto.

La torta 3 seleccionada por los degustadores presentó la composición de harinas leche 2:2:2, con un porcentaje de yacón de un $15.7 \%$ y cubierta de mora-curuba, en la selección de la mezcla fue la que más dificultades exhibió para el logro de las características en la textura del producto, lo que obligo a la realización de 10 pruebas hasta obtener la mejor comportamiento con el $48 \%$ en la composición de harinas - leche, sin embargo, se recomienda desarrollar nuevas pruebas hasta la optimización de la calidad de la miga típica de este tipo de producto.

La evaluación sensorial permitió establecer la aceptación del dulzor de los productos elaborados con yacón y rectificados con sucralosa, en galleta 2, pan 3 y torta 3 , por los degustadores de mayor edad.

Los resultados finales muestran que los grados de aceptación promedio de la evaluación sensorial corresponden al rango de valores $6.0-7.0$, correspondientes a juicios gustó moderadamente, gustó ligeramente, información que permite continuar con las pruebas, mejorando las formulaciones para elaboración de productos que puedan competir en el mercado.

Los degustadores no entrenados con edades entre 25 - 35 años representaban el 32\% del total de la población, dando a los tres tipos de productos los máximos puntajes, lo que permite inferir, que es muy probable que los productos sean aceptados en el mercado por este segmento de la población.

Los degustadores entre 19 y 24 años, presentaron una aceptación moderada de los productos, recomendando en sus observaciones la mejora del color de los productos con solo mora o curuba, pero además aumentando el dulzor de los productos finales.

Los degustadores con menor aceptación de los productos se encontraron en el segmento de edades entre 15 - 18 años con un porcentaje del $23 \%$, recomendando un aumento en el dulzor de los productos y mejorando el color de los productos finales.

Los productos elaborados galleta 2, pan 3 y torta 3 tienen en su composición fructooligosacáridos o fructanos presentes en el yacón, que al no ser metabolizados directamente por el tracto digestivo humano, pueden desplazarse en casi todo su recorrido por el tracto digestivo sin ser modificados, comportándose como un ingrediente prebiótico que estimula el crecimiento y la actividad de bacterias propias del intestino. 
Formulación y elaboración de productos de panificación con yacón (Smallanthus sonchifolius) como endulzante, para la población con deficiencias en el metabolismo de los disacáridos

Los productos elaborados galleta 2, pan 3 y torta 3, por tener en su composición fructooligosacáridos, componentes sustitutos hipocalóricos de varios tipos de azúcares, pueden ser incluidos en los regímenes dietéticos para bajar de peso y representan una alternativa en el diseño de dietas hipocalóricas para diabéticos especialmente, sin embargo, pueden ser consumidos por toda la población.

Las dificultades presentadas en la caracterización de la masa para torta a partir de diferentes composiciones de harinas sugieren, una reformulación del producto con el fin de mejorar la textura del resultado final.

\section{REFERENCIAS}

Alfaro D G 2004, Principales resultados de los últimos 10 años. Programa de Alimentos y alimentos naturales, Umss, Cochabamba.

Bernal, S L 2014, 'Una visión del sistema de certificación en inocuidad de los alimentos Fssc22000-1', Revista Publicaciones e Investigación, 8, 151-159

Boyle, J P, Honneyeutt A A, Narayan, K M, Hoerger, T J, Geiss, L S, Chen, H \& Thompson, T J 2001, 'Projections of diabetes burden though 2050: impactof changing demography and discase prevalence in US', Diabetes Care, 24 (11), 1936 - 1940.

Bruggebcate, S J, Bovee-Oudenhoven, I M, Lettink-Wissink, M L, Katan, M B \& van der Meer, R 2006, 'Dietary fructooligossaccharides affect intestinal barrier function in health men' Journal of Nutrition, 136 (1), 70-74

Chen, A, Resurrección, A \& Paguio, L 1996, 'Age appropiate hedonic scales to measure food preferences of Young children', Journal of Sensory Studies, 11 (2), 141 - 163.

Delgado, J 2016, 'Aplicación del ultrasonido en la industria de alimentos', Revista Publicaciones e Investigación, 6, 141-152.

Díaz, F, Lizarazo, A \& Torres O 2016, 'Determinación de la huella hídrica del algodón', Revista Publicaciones e Investigación, 10, 23-37.

Giraldo F, Vargas, T \& Gil, H 2016, 'Mejoramiento del proceso de deshidratación de uchuva', Revista Publicaciones e Investigación, 3, 37-49.

González, A, Amarillo, G, Amarillo, M \& Sarmiento, F 2016, 'Drones aplicados a la agricultura de precisión', Revista Publicaciones e Investigación, 10, 39-46.

Gordillo, R 2013, 'Efecto hipoglicemiante del extracto acuoso de las hojas de Smallanthus sonchifolius (yacón) en pacientes con diabetes melitus tipo 2, Tesis Facultad de Farmacia y Bioquímica', Universidad Nacional de San Marcos, Lima-Perú.
Huaycho, H, Aruquipa, R, Mercado, G, Trigo, R, Bosque, H \& Condori, J 2016, 'Conocimientos tradicionales en Yacón o aricoma (Smanllantus sonchifolius) en comunidades de Mocomoco', Coroico e Irupana de La Paz, vol. 3, no. 2, consultado: 15 septiembre 2017, http://www.revistasbolivianas.org.bo/scielo. php?pid=\$2409

Institute of Food Technologists (IFT) 2013, What are fructooligossacharides and how do they provide digestive, immunity and bone health benefits? [Página web], consultado: 15 septiembre 2017, https://www.sciencedaily.com/ releases/2013/07/130716115728.htm

Lachman, J, Fernandez, E, \& Orsak, M 2003, 'Yacón [Smallanthus sonchifolius (Poepp. Et Endl) H.Robinson] Chemical composition and use-a review', Plant Soil Environ, 49 (6), 283-290.

López, T 2007, 'Valorización de la raíz de yacón: obtención de un jarabe rico en fructooligosacáridos', Revista Investigación y Desarrollo, (7), 93-106.

Madrigal, L \& Sangronis, E 2007, 'La insulina y derivados como ingredientes claves en alimentos funcionales', Archivos Latinoamericanos de Nutrición, 57(4), 387-396.

Manrique, I, Párraga, A, \& Hermann, M 2005, Jarabe de yacón: principios y procesamiento. Serie: Conservación y uso de la biodiversidad de raices y tubérculos andinos: Una década de investigación para el desarrollo (1993-2003), Centro Internacional de la Papa, Universidad Nacional Daniel Alcides Carrión, Fundación Erbacher, Agencia Suiza para el Desarrollo y la Cooperación, Lima.

Mansilla, R, López, C, Flores, M \& Espejo, R 2010, 'Estudio de la biología reproductiva en cinco accesiones de Smanllanthus sochifolius (Poepp \& Endl) mediante técnicas de polinización controladas', Ecología Aplicada, 9 (2), 73-78.

Margalef, M, Gómez, M, Jiménez, M, Tóffoli, S, Valdez, G, Castillo, F, Estrada, L, Argenti, P, Bonomo, A, Bonfiglio, G, Burgos, V, Jury, A \& González, L 2013, Ingredientes no tradicionales para la formulación de alimentos funcionales, Facultad de Ingeniería, Consejo de Investigación Universidad Nacional de Salta, Argentina.

Ministerio de Salud y Protección Social 2017, Portal institucional [Página web], consultado: 11 agosto 2017, https://www. minsalud.gov.co/Paginas/Al

Pedreschi, R, Campos, D, Noratto, G, Chirinos, R \& CisnerosZevallos, L 2003, 'Andea yacon roots Smallanthus sonchifolius (Poepp\&endl) Fuctoologosaccharides as a potencial novel source of prebiotics', Journal of Agricultural and Food Chemistry, 51 (18), 5278-5284

Quintana, F, Gelvez, P \& Mendoza, L 2014, 'Estandarización de la fase de Fermantación I en la obtención de licor de mandarina utilizando levadura "Saccharomyces Cerevisiae", Revista Especializada en Ingeniería, (8), 139-149.

Sánchez, B, Villareal, R \& Torres, O 2014, 'Estimación de la huella hídrica para el cultivo de pitahaya amarilla (Selenicereus megalanthus)', Revista Publicaciones e Investigación, 9, 135-146 
Sánchez, S \& Genta, S 2007, 'Yacón: un potencial producto natural para el tratamiento de la diabetes', Boletin Latinoamericano y del Caribe de Plantas Medicinales y Aromáticas, 6 (5), 162 - 164.

Sangeethaa, P, Ramesha, M \& Prapullaa, S 2005, 'Recent trends in the microbial production, analysis and application of fructooligosaccharides', Trends in Food Science and Techonology, 16 (10), 442-457

Seminario, J, Manrique, I \& Valderrama, M 2003, El yacón: Fundamentos para el aprovechamiento de un recurso promisorio, Centro Internacional de la Papa (CIP), Universidad Nacional de Cajamarca y Agencia suiza para el Desarrollo y la Cooperación (Cosude), Lima - Perú.

Sendaya, D 2013, ‘QQué es el control predictivo y hacia donde se proyecta?', Revista Publicaciones e Investigación, 7, 53-59

Sitonio, E \& Menegalli, F 2013, 'Cubiertas y películas comestibles aditivos bioactivos para la conservación de la fruta', RRevista Publicaciones e Investigación, 6, 12-22

Takaneka, M, Yan, X, Ono, H, Yoshida, M, Nagata, T \& Nakanishi T 2003, 'Caffeic acid derivatives in the roots of yacon (Smallantus sonchifolius)', Journal of Agricultural and Food Chemistry, 51 (3), 793-796
Tamayo, M 2005, 'El proceso de la investigación científica, incluye evaluación y administración de proyectos', en M. Tamayo, El proceso de la investigación científica, Limusa, México D.F.

Tiwari, A \& Rao, J 2002, 'Diebetes mellitus and multiple therapeutic approaches of phytochemicals: present status and future prospects', Current Science, 83(1), 30-38

Valderrama, M \& Manrique, I 2003, Yacón: Fundamentos para el aprovechamiento de un recurso promisorio. Centro Internacional de la Papa, Lima-Perú.

Valderrama, M 2005, Manual del cultivo del yacón. Experiencias de introducción y manejo técnico en el valle de Condebamba, Programa Pymagros (Convenio Cosude - Minag), Cajamarca- Perú.

Valdez, C, Margalef, M, \& Gómez, M 2003, 'Formulación de barra dietética funcional prebiótica a partir de harina de yacón’, Diaeta, 31 (142), 27-33.

Yan, X, Suzuki, M, Ohnishi-Kameyama, M, Sada, Y, Nakanishi, T \& Nagata, T 1999, 'Smallanthus sonchifoluis: generalities of the fruit', Journal of Agricultural and Food Chemistry, 47(11), 4711-4713 
